\title{
Wi-Fi technology - an uncontrolled global experiment on the health of mankind
}

\author{
Marko Markov ${ }^{1} \&$ Yuri G. Grigoriev ${ }^{2}$ \\ ${ }^{1}$ Research International, Williamsville, NY, USA, and ${ }^{2}$ Russian National Committee of \\ Non-lonizing Radiation Protection, Moscow, Russia
}

The twenty-first century is marked with exponentially increasing development of technologies that provide wireless communications. To the pollution of the atmosphere with radio and TV signals, not only satellite communications but also any varieties of the Wi-Fi networks are added. By 2010 in the USA, 285 million mobile phone subscribers have been registered (for a little bit more than 300 million inhabitants). The estimate for the world is more than 5 billion mobile phone users at approximately 7 billion people living on this planet. Approximately 2 years ago, the International Agency of Research on Cancer (IARC) classified the electromagnetic fields used in mobile communication as a possible cancerogene. This paper discusses the potential health hazard and lack of scientific assessment and regulatory actions in protection of the life on the planet.

Keywords: WiFi, pollution, hazard, Radiofrequency electromagnetic fields

\section{The problem: lonizing versus nonionizing radiation}

Contemporary science is increasingly using and investigating two physical factors such as ionizing and nonionizing radiation, with an attempt $t$ ? search for common mechanisms of action and evaluation of the public benefit and health hazard. What is common here is the word "radiation." However, from the viewpoint of physics, these are two different factors that might be found in an environment. Importantly, they act simultaneously, but are discussed separately, entirely neglecting the existing background of the other factor.

It has been well established that ionizing radiation usually provokes effects based on energetic mechanisms and ionization of tissues. This action is characterized with threshold levels and could develop within short time after irradiation. Speaking on ionizing radiation, scientists and public health experts, based on decades of investigation, have come to know about a large variety of unfavorable, potentially harmful effects that developed hours (sometimes days) after irradiation. This was well confirmed in the evaluation of health effects and care for personnel and population after Chernobyl accident a quarter of century ago (Grigoriev, 2012a,b; Sage, 2012). Throughout the world, interest was also excited by the recent Fukushima disaster in March 2011.

Correspondence: Marko Markov, Research International, Williamsville, NY, USA

E-mail: msmarkov@aol.com 
Shortly speaking, for ionizing radiation situation might be summarized as follow:

- radiobiology set lines of standards for limited exposure to ionizing radiation,

- research and monitoring of ionizing radiation,

- mapping of natural sources of ionizing radiation (IR),

- use of radioactive isotopes in medicine and technology.

Of course, everything here is in "normal" condition and is not related to potential and occurred catastrophes. Do we know enough about ionizing radiation? Probably not. However, there is a continuous improvement in standards and monitoring methods and technology developed by radiobiology and industry.

What do we know about nonionizing radiation? Basically NOTHING. Even the simplest and longer studied behavior of natural magnetic, electric and electromagnetic fields (EMFs) is far of complete knowledge. On the other hand, the planet is exposed to various EMFs with space origin. The most sophisticated analysis of the evolution of biosphere from the viewpoint of space-earth relationships was done by Chizevskii long time ago. More than 45 years ago, a brilliant Soviet magnetobiologist Kholodov (1976) wrote a book "Man in the magnetic web." Long before the occurrence of mobile communications, Kholodov pointed out that the entire biosphere is immersed in the ocean of the electromagnetic waves.

However, this is just a part of the problem. The fast development of satellite communications, followed by wireless communications and recently Wi-Fi technology dramatically, changes the electromagnetic environment. To continuous action of complex and unknown (by sources, amplitudes, frequencies) electromagnetic fields is exposed entire biosphere and every organism living on this planet. We usually neglect this complex that includes radio and TV transmissions, satellite signals, mobile phones and base stations and wireless communications.

Speaking on the potential hazard of Wi-Fi technologies, one should not forget that it includes not only mobile phones but also more importantly all means of emitters and distributors of Wi-Fi signals, mainly antennas, base stations and satellites. In many public locations, own systems are introduced in order to facilitate the work performance. Well, this might be understood. However, why Wi-Fi communications are secured in the subway tunnels? It obviously requires high and oriented power to which are exposed all passengers in the trains. Just to make comfortable the users of mobile phones or other WiFi gadgets.

The search for specificity of the Wi-Fi technology can point to the following:

- Popular technology that allows an electronic device to exchange data wirelessly (using radio waves) over a computer network, including high-speed Internet.

- A device that uses Wi-Fi can connect to a network resource such as the Internet via a wireless network access point. Such an access point has a range of about $20 \mathrm{~m}(65 \mathrm{ft})$ indoors and a greater range outdoors.

As a result,

- brains of 7,000,000,000 people are exposed to unknown spectrum of EMF,

- there are no criteria for hazard,

- no monitoring,

- no research and

- no prevention.

Generally speaking, we do not know if or to which extent the Wi-Fi radiation alters the physiology of normal, healthy organisms. The situation became more complex 
when we are asking about the influence on children, on aging adults or sick individuals. Especially for children we should point that:

- children are exposed to all spectrum of EMF that polluted the biosphere,

- who is brave enough to say that Wi-Fi cannot influence the children's brain which is still underdeveloped,

- children are prone to electronic toys - cell phones and wireless games.

\section{EMFs in biosphere}

For the nonionizing radiation very rarely, one could never say that effects can be seen during irradiation or shortly after it and do not develop in similarity with the effects of ionizing radiation. Even when "thermal" mechanisms and specific absorption rate (SAR) approach are considered, necessity of certain time was also recognized to develop and manifest effects (Markov, 2006).

Our long experience in radiobiology and magnetobiology allows us to affirm that biological effects of EMFs are nonthermal and have to be discussed from the lowenergy considerations, such as conformational changes in biological structures, effects on signal-transduction cascade, as well as in their manifestations on the level of important biomolecules. More likely, the effects of EMF have informational character and because of that it needs certain time to manifest itself and initiate further changes in biochemistry and physiology (Markov, 2012).

After 1976, two serious problems immersed in the public health discussion - potential hazard of low-frequency EMF from power and distribution lines (in the late 1970s and 1980s) and mobile communications (mobile phones, Wi-Fi Internet and base stations) that utilize high-frequency EMF and represent a serious hazard for public health today. One of the authors stressed, today the mankind participates in a global experiment conducted by the industry without regulation, rules and control (Grigoriev, 2012b).

During the last two decades, wireless communications (cell phones, Internet and Wi-Fi technology) became the fastest developing technology that spread around the world. It is close to 5 billion mobile phones registered in both developed and developing countries. In addition to the users of these technologies, the entire world population is exposed to exponentially increasing radiofrequency (RF) radiation from base stations and satellite antennas.

Being in public space, such as schools, transportation, supermarkets and hospitals, one may see that nearly any young person is caring or handling electronic device from portable electronic games to the last edition of smart phone. This is what contemporary technology and industry offered as an advance of civilization. The question is "At what price?"

The cellular telephone delivers a power density of RF radiation about 2 billion times greater than that occurring naturally in the environment. Since the mobile phones are designed to operate at the side of the user's head, a large part of the transmitted energy is radiated directly into that person's brain. The absorbed energy potentially could cause dangerous and damaging biological effects within the brain. The small cellular telephones effectively deposit large amount of energy into small areas of the user's head and brain.

\section{The problem of EMF emission and absorption}

It should be reasonable to mention that this problem, which has aggravated by the time, is not new. In 1995, Kane stated "Never in human history has there been such a 
practice as we now encounter with the marketing and distributing of products hostile to the human biological system by an industry with foreknowledge of those effects". What may be added to this powerful statement - R. Kane was high level Motorola executive.

One of the first papers on the absorption of electromagnetic energy was published by Schwan \& Piersol (1954), in which they connected this absorption with the tissue composition. It is important to note that tissue composition is a very complex one and varies from organ to organ from person to person. From biophysics point of view, the energy absorption also depends on the depth of penetration for the specific frequency range (for $825-845 \mathrm{MHz}$ the penetration depth into the brain tissue is from 2 to $3.8 \mathrm{~cm}$ ) (Polk \& Postow, 1986).

Forty years ago, Michaelson (1972) wrote

It should be understood that a cumulative effect is the accumulation of damage resulting from repeated exposures each of which is individually capable of producing some small degree of damage. In other words, a single exposure can result in covert thermal injury, but the incurred damage repairs itself within a sufficient time period, for example hours or days, and therefore is reversible and does not advance to a noticeable permanent, or semi-permanent state. If a second exposure or several repetitive exposures take place at time intervals shorter than that needed for repair, damage can advance to a noticeable stage.

In other words, the repeated irritation of a particular biological area, such as a small region of the brain, can lead to irreparable damage. Given the existence of energy absorption, "hot spots" and then each damaging exposure to RF radiation provide a new opportunity that the damage will become permanent. Part of the problem is that an exposed person would never know of the penetration and damage.

As seen above, the basic science from the 1950s to 1990s reported evidence that high-frequency EMF can have harmful effects on human organisms especially on human brain. It has been even detailed with respect to the frequency range: 900 $2500 \mathrm{MHz}$. Number of studies had pointed out that electromagnetic energy in the $900 \mathrm{MHz}$ region may be more harmful because of its greater penetrating capability compared to $2450 \mathrm{MHz}$; therefore, more energy in the $900 \mathrm{MHz}$ frequency range is deposited deeply within the biological tissue. In 1976, Lin concluded that $918 \mathrm{MHz}$ energy constitutes a greater health hazard to the human brain than does $2450 \mathrm{MHz}$ energy for a similar incident power density (Lin, 1976).

Let us remind that studies of diathermy applications consistently show that electromagnetic energy at frequencies near and below $900 \mathrm{MHz}$ is best suited for deep penetration into brain tissue. The depth of penetration is noticeably greater at this frequency range, which includes the portable cellular phone frequencies as compared with higher frequencies. Of importance it was also proven that deep tissue heating is obtained without detecting significant heating in the surface tissues. By their nature, the frequencies that provide the best therapeutic heating would also be the frequencies that could be most hazardous to man in an uncontrolled situation. High absorption occurs in the inner tissue such as the brain while fat and bone absorption is many times less (Johnson \& Guy, 1972).

Since the human brain has little, if any, sensory capability, damage or trauma occurring internally will not be felt until the effects, such as heating, are so severe that they work their way outward. If tissue damage occurs within a localized region of the brain it may be completely unnoticed. The threshold for irreversible skin damage is about $45 \mathrm{C}$ which is also the temperature at which pain is felt. So, by the time a person, exposed to RF radiation, feels pain at the skin that skin is irreversibly 
damaged, as is the deeper tissue beneath the skin. Similarly, internal heating of the brain tissue would not be sensed as a burning sensation. Likely, there would be no sensation at all. Interest in the ability to "sense" the presence of high levels of RF radiation motivated researchers to determine threshold levels for detecting heat sensations due to radiation exposure (Justensen, 1982).

An obvious question arises: if science had developed such knowledge decades ago why today these questions are not at the priority list for research? We would point here two major reasons: the political power of the industry and the failure of the scientific community.

The major standards and guidelines established by the engineering community, Institute of Electrical and Electronic Engineers (IEEE) in 2005 and International Commission on Non-Ionizing Radiation Protection (ICNIRP) in 2009, provide approach and terminology which are not accepted by physics and biological communities, but nevertheless remain the guiding rules (mainly for the industry). One can only wonder how is it possible to speak about potential "health effects" of RF instead of "health hazard." The misuse of the term "health effect" is probably done on purpose not to alarm the general public about the hazard of the use of microwave radiation in close proximity to the human brain.

It has been pointed elsewhere (Markov, 2006) that the engineering committees that state "Nonthermal RF biological effects have not been established" basically are guiding science and society into wrong direction. Declining the possibility of nonthermal effects is not reasonable, but more important is that they mixed "effect" and "hazard." In magnetobiology literature, hundreds of papers have been published discussing the genotoxicity and modifications in DNA and other important biological molecules. In the paper of Israel, Zaryabova, and Ivanova (2013), it was correctly pointed out that even the definition of the thermal effect by these international bodies is not accurate.

The epidemiological teams claim that "there is no consistent evidence for the occurrence of the modification." They also state that "there is no conclusive and consistent evidence that nonionizing radiation emitted by cell phone is associated with cancer risk" (Boice \& Tarone, 2011). It is remarkable that this paper was published after International Agency of Research on Cancer (IARC) defined RF as "possible cancerogenic for humans."

In a recent paper (Markov, 2012), we discussed the fact that the long-delayed publication of the INTERPHONE data resulted in the strange situation: two groups of participants in the project published two papers that basically contradict each other. Another critical point of consideration is the fact that the energy absorption characteristics that make the 750 and $915 \mathrm{MHz}$ frequencies so desirable for hyperthermia and diathermy treatments have the very same absorption characteristics that make the $825-845 \mathrm{MHz}$ cellular telephone transmit band so dangerous (Kane, 1995).

For more than half a century, a very serious group of policy-makers and even scientists are playing around the term SAR. The SAR given in terms of Watts per kilogram $(\mathrm{W} / \mathrm{kg})$ or milliwatts per gram $(\mathrm{mW} / \mathrm{g})$ is assumed to provide a measure of absorbed energy in a given tissue. Absorption, not delivery. This term is particularly advantageous for the industry since the energy absorption in biological bodies and specific organs is nonuniform and frequency dependent. However, until today SAR is more often used to describe the energy delivered by the source of the EMF. One can only wonder how a device may be characterized by the SAR. To repeat, the SAR identifies the amount of energy that is absorbed in a gram of tissue.

The inappropriate use of the SAR leads proponents of Wi-Fi technology to affirm that since there is no heating of brain by RF EMF, there is no hazard for the human 
brain, completely neglecting the fact that most of the biological effects are nonthermal. We are convinced that the safety standards would need to be restated in terms of internal energy absorption.

\section{Wi-Fi and mobile communications effects on children}

To the large range of high-frequency electromagnetic fields is exposed any age group from babies to very old people, most of which do not have interest toward mobile communications. This is extremely dangerous especially for children who use mobile phones from early age without any control from the site of their parents.

In evaluation of the mobile phone EMF hazard for children, the main problem is the direct effect of the EMF on the brain of the child, which is the critical organ. One should remember that the child's brain is still in process of the physiological development. Moreover, using the mobile phones children expose to EMF daily and in duration of years not only brain, but the complex nerve structures in the internal year that are responsible for normal functioning of the hearing and of the vestibular analyzers (Grigoriev, 2006a,b, 2012b).

We discuss the basic principles for the estimation of the hazard of microwave EMF for this part of population who most frequently use contemporary modalities of the wireless communications - children and young adults.

- For the first time in the history of our civilization, the most critical systems in human organism - brain and the nerve structures - inside the internal year of children and adults are exposed to complicated and unknown exposure to EMF.

- Due to physiological reasons, the brain of a child exhibited higher rates of specific absorption of EMF compared with adult's brain. Thus, more compartments of the brain are exposed to high-frequency EMF, including those responsible for intellectual development. Recently, data about unfavorable influence of the EMF on the cognitive function of the brain have been published (Grigoriev, 2012b).

- Once again, children's brain is in the stage of physiological development and formation of the analytical function could be inhibited, including the development of pathological functions, such as epileptic syndrome (Grigoriev \& Sidorenko, 2011).

- Children became owners of the mobile phones and other uncontrolled sources of EMF.

- Due to the age specificity, children cannot evaluate the potential hazard of the mobile phone. Rather they consider it as a wonderful tool for communication and entertainment.

- Taking into account the technological development in the wireless communications, it will be fair to say that children at the age of $3-4$ will be using mobile communication longer than their parents with respect to their entire life. There is evidence that today the children and young adults are the most aggressive users of mobile communications and Wi-Fi technology.

- It is unfortunate that with support and encouragement of parents these two groups are exposed for long hours to uncontrolled influence of EMF in RF range.

In addition, as shown by Divan, Kheifets, and Obel (2008), today one cannot neglect the fact that even during the embryonic development the child organism could be exposed to electromagnetic influence.

It is reasonable to remind the position of the WHO "CHILDREN ARE DIFFERENT FROM ADULTS." Children have a unique vulnerability. As they grow and develop, 
there are "windows of susceptibility": periods when their organs and systems may be particularly sensitive to the effect of certain environmental threats (WHO, 2003, 5p.).

Unfortunately, there is lack of scientific data and analysis of the estimation of the potential hazard of mobile communications. Nobody investigates thoroughly the potential damages in the brain of children, which potentially could occur in their developing brain (Markov, 2012). Studies of potential of development of the long-range modification of the brain functions in children whose brain have been exposed to continuous irradiation with high-frequency EMF are completely absent. Therefore, we should start from zero. At the same time, international and governmental agencies responsible for standards are slow in responding to the exponential growth of technologies and in principle completely neglect the hazard for children (Grigoriev, 2008; Markov, 2012).

More than 10 years ago, at the WHO organized meeting on harmonization of standards one of the authors pointed out that neglecting the hazard of highfrequency EMF for children is a crime against humanity (Markov, 2001).

It should be stressed that at ionizing radiation the dose/effect dependence is present in any case of consideration, while with nonionizing radiation the threshold effect is basically absent, the occurrence of bioeffects needs some time to develop and might be diminished or enlarge by modulation (Grigoriev, 2006,a,b).

It is unfortunate that until now scientists, politicians and lawmakers underestimate the unfavorable effects on human health that may occur as a result of long, continuous exposure to nonionizing radiation (often exposure from different factors). The other problem arises from the engineering community, which forces the notion that nonthermal effects are only of importance.

We believe that scientific community must be more insisting in comprehension of potential hazard that mobile exchange of information is potentially invoked in human organisms. In that aspect, it is very important that the IARC classified the RF EMF as a possible cancerogene (2B). It is important to note that for less than two years this major International Agency switched from "no conclusive evidence for health hazard" to "possible cancerogene". At the same time, ICNIRP continues to affirm that "the trend in the accumulating evidence is against the hypothesis that mobile phones can cause brain tumors in adults" (Boice \& Tarone, 2011).

A question eventually arises "Why ICNIRP takes this position?" We may offer two different, but eventually complimentary answers: first, most of the funding for ICNIRP is coming from industry and second, eventually more important - most of the members of ICNIRP are engineers for whom only thermal effects may cause biological effects.

It is time that scientific community in general as well as radiobiologists and magnetobiologist rings the bell that it is time to recognize and estimate the potential hazard for human health of the increasingly elevated background radiation levels.

In 2003, IEEE published a standard that affirmed that biological effects can only be thermal (Cho \& D'Andrea, 2003). No heat - no effect. This policy serves very well in the industry and creates a serious barrier preventing biology-based assessment of the human health.

The scientific community must be more insisting in comprehension of potential hazard that mobile exchange of information potentially invoke in human organisms. In addition, the development of nuclear medicine and nuclear energy elevates the radiation background over the planet, not even considering Chernobyl and Fukushima disasters (Akahane et al., 2012; Grigoriev, 2012c). 
In conclusion, we would like to point out the following:

1. For a number of reasons, the evaluation and prevention of hazard from ionizing radiation have been developed during the last 60 years.

2. The problem of potential hazard of nonionizing radiation is studied for significantly shorter period of time and significant differences in standards in different countries can be seen. It is due to the fact that in North America the standards are based on engineering computation, while in former Soviet Union and Easter Europe the standards are biologically based. Despite all efforts of the WHO for harmonization of standards, today standards do not consider the real pollution of the environment with nonionizing radiation.

3. The comparison of both sources of radiation leads to conclusion that the continuous noncontrolled exposure of the entire civilization to low-intensity EMFs represents now more serious problem for the mankind than ionizing radiation where the sources of radiation are under strict control and are well localized.

4. For the first time in the history of mankind, because of aggressive use of mobile phones children are exposed to harmful nonionizing radiation and potentially are subject of larger risk than adults. Even if the dose received by the children's brain is the same as for adults, due to the specificity of the body size and physiological development, children are in greater danger. It might be compared to the hazard of EMF for professional groups.

5. In early 2012, the European Parliament voted by 512 to 16 to urge member countries to impose stricter limits for exposure to radiation from mobile phone and Wi-Fi technologies, especially taking care for the most sensitive population of children.

6. The scientific and medical communities are obliged to ring the bell - the health and standardization institutions must urgently develop recommendations and actions for protection of the civilization and especially children.

7. We should stop telling the science, politicians and general population that Wi-Fi is harmless.

8. We should better be honest and say that "we do not know what long-term effects might be."

\section{Who else if not we? When if not now?}

\section{Declaration of interest}

The authors report no conflicts of interest. The authors alone are responsible for the content and writing of the paper.

\section{References}

Akahane K, Yonai S, Fukuda S, Miyahara N, Yasuda H, Iwaoka K, Matsumoto M, et al. (2012). The Fukushima nuclear power plant accident and exposure in the environment. Environmentalist 32(2): 136-143.

Boice J, Tarone RE (2011). Cell phone, cancer and children. J Natl Inst Cancer 103(16):1211-1213.

Cho CK, D'Andrea JA (2003). Review of effects of RF fields on various aspects of human health. Bioelectromagnetics 24:S5-S6.

Divan H, Kheifets L, Obel C (2008). Prenatal and postnatal exposure to cell phone use and behavioral problems in children. Epidemiology 19(4):523-529.

Grigoriev Yu (2006a). Mobile telecommunication: Radiobiological issues and risk assessment. Proc Latvian Acad Sci B 60(1):6-10.

Grigoriev Yu (2006b). Development of electromagnetic field somatic effects: Role of modulation. Proc Latvian Acad Sci B 60(1):11-15. 


\section{M. Markov \& Y.G. Grigoriev}

Grigoriev Yu (2008). Russian NCNIRPG and standards. New condition of EMF RF exposure and guarantee of population health. Proceedings of the International Conference "EMF and Health - a Global Issue", London.

Grigoriev Yu, Sidorenko A (2011). Nonthermal electromagnetic fields and estimation of the probable development of the convulsive syndrome. Biophysics 56(2):351-357.

Grigoriev YG (2012a). Cellular communications and public health. Radiat Biol Radioecol 52(2):1-4.

Grigoriev YG (2012b). Mobile communications and health of population: the risk assessment, social and ethical problems. Environmentalist 32(2):193-200.

Grigoriev YG (2012c). Six first weeks after Chernobyl nuclear accident. Environmentalist 32(2):131-135.

Hygienic requirements for the siting and operation of land mobile radio communication equipment. SanPiN 2.1.8/2.24.1190-03. Approved by the Chief Government Sanitary Physician of the Russian Federation on 31 January 2003. Moscow, Russian Federation (in Russian).

IARC WHO, Classifies radiofrequency electromagnetic fields as possibly carcinogenic to humans. Press release no. 208, 31 May 2011, 3 p.

IARC (2011). Carcinogenicity of radiofrequency electromagnetic fields. Lancet Oncol 12(7):624-625.

Israel M, Zaryabova V, Ivanova M (2013). Electromagnetic field occupational exposure. Non-thermal vs. thermal effects. Electromagn Biolo Med (in this issue).

Kane R (1995). Cellular telephone Russian roulette. New York: Vantage Press, Inc. p 241.

Kholodov YA (1976). Man in magnetic web. Moscow: Nauka.

Lin JC (1976). Interaction of two cross-polarized electromagnetic waves with mammalian cranial structures. IEEE Trans Biomed Eng BME 23(5):371-375.

Markov MS (2001). Magnetic and electromagnetic field dosimetry - necessary step in harmonization of standards. - Proceedings of WHO Meeting, Varna, April 2001. Available at http://www.who.int/ peh-emf/publications/Varna

Markov MS (2006). Thermal vs. nonthermal mechanisms of interactions between electromagnetic fields and biological systems. Bioelectromagnetics: current concepts. Dodrecht, The Netherlands: Springer. pp $1-16$.

Markov MS (2012). Cellular phone hazard for children. Environmentalist 32(2):201-209.

Michaelson SM (1972). Human exposure to nonionizing radiant energy - potential hazards and safety standard's. Proceedings of the IEEE 1:389-421.

Polk C, Postow E, editors. (1986). CRC handbook of biological effects of electromagnetic fields. Boca Raton, FL: CRC Press.

Sage C (2012). The similar effects of low-dose ionizing radiation and non-ionizing radiation from background environmental level of exposure. Environmentalist 32(2):144-156.

Schwan HP, Piersol GM (1954). The absorption of electromagnetic energy in body tissues. Int Rev Phys Med Rehabil 33(6):371-404.

WHO, Backgrounder no. 3, April 2003, 3 p. 